\title{
Deterministic Delay Bounds for VBR Video in Packet-Switching Networks: Fundamental Limits and Practical Tradeoffs
}

\author{
Dallas E. Wrege, Edward W. Knightly, Hui Zhang, and Jörg Liebeherr
}

\begin{abstract}
Compressed digital video is one of the most important traffic types in future integrated services networks. However, a network service that supports delay-sensitive video imposes many problems since compressed video sources are variable bit rate (VBR) with a high degree of burstiness. In this paper, we consider a network service that can provide deterministic guarantees on the minimum throughput and the maximum delay of VBR video traffic. A common belief is that due to the burstiness of VBR traffic, such a service will not be efficient and will necessarily result in low network utilization. We investigate the fundamental limits and tradeoffs in providing deterministic performance guarantees to video and use a set of 10 to 30 minute long MPEG-compressed video traces for evaluation. Contrary to conventional wisdom, we are able to show that, in many cases, a deterministic service can be provided to video traffic while maintaining a reasonable level of network utilization. We first consider an ideal network environment that employs the most accurate deterministic, time-invariant video traffic characterizations, the optimal Earliest-Deadline-First packet schedulers, and exact admission control conditions. The utilization achievable in this situation provides the fundamental limits of a deterministic service. We then investigate the utilization limits in a network environment that takes into account practical constraints, such as the need for simple and efficient policing mechanisms, packet scheduling algorithms, and admission control tests.
\end{abstract}

\section{Introduction}

Future packet-switching integrated services networks must support applications with diverse traffic characteristics and performance requirements. Of the many traffic classes in integrated services networks, delay-sensitive Variable Bit Rate (VBR) video traffic poses a unique challenge. Since the required service is delay-sensitive, the network must support a resource reservation scheme [4] that allocates network resources for each VBR video stream. However, the burstiness of VBR video traffic makes it difficult to determine the amount of resources required. On one hand, if resources are reserved according to the average rates of the VBR video sources, unacceptable delays or packet losses may result when the sources are transmitting near their peak rates. On the other hand, if resource reservations are based on sources' peak rates, the network may be underutilized most of the time.

D. E. Wrege and J. Liebeherr are with the Department of Computer Science, University of Virginia, Charlottesville, VA 22903. Their research is supported in part by the National Science Foundation under Grant No. NCR-9309224

E. W. Knightly is with the Department of Electrical Engineering and Computer Sciences, University of California at Berkeley, Berkeley, CA 94720. His research is supported in part by Sandia National Laboratories.

H. Zhang is with the School of Computer Science, Carnegie Mellon University, Pittsburgh, PA 15213.
In this study, we investigate utilization limits achievable in packet-switching networks with deterministic service guarantees to delay-sensitive VBR video traffic. A deterministic service ensures that no packets are dropped or delayed beyond their guaranteed delay bound. Such a service requires admission control tests to determine if the network has sufficient resources to support a new connection without degrading the service of existing connections $[4,7]$. The tests for a new connection are based on a characterization of the expected traffic for the new connection. If accepting or admitting the new connection may result in violations of service guarantees for any existing connection, the connection is not established. For admitted connections, the network performs traffic policing, that is, it ensures that the admitted connections adhere to the traffic characterization given to the network for the admission control tests. If a connection's traffic is policed by the network, the traffic in excess of its specified characterization may not be permitted to enter the network.

A key challenge of incorporating VBR video traffic into networks with service guarantees lies in the difficulty of finding an appropriate traffic characterization that captures the dynamics of the source. A rich set of literature exists on characterizing VBR video traffic by a stochastic process using models such as Markov-modulated, Auto Regressive, TES, and Self-similar (see [5, 10] and the references therein). While such stochastic models of a source have the advantage that they may be used to potentially achieve higher network utilization by exploiting the statistical properties of the sources, they have several important disadvantages. First, it is difficult to implement a policing mechanism that enforces a stochastic traffic characterization. Moreover, most stochastic models for characterizing video traffic are either not powerful enough to capture the burstiness and the timely correlations of a video source, or they are too complex for practical implementation [10]. Finally, since stochastic approaches to VBR traffic characterization provide only statistical guarantees, they cannot be applied to deterministic network services $[8,9,17]$.

For such reasons, in this work we consider only deterministic traffic models that characterize VBR traffic in terms of a worst-case description. It is a common belief that such a worst-case approach necessitates allocation of network resources according to sources' peak rates ${ }^{1}$, necessarily leading to low network utilization for bursty traffic. However,

\footnotetext{
${ }^{1}$ For VBR video, we consider the stream's peak rate to be the size of the largest video frame divided by the inter-frame time.
} 
several recent works show that this conventional wisdom is not correct and that peak-rate allocation is not required even for providing deterministic service $[2,8,9,17]$.

In this study, we analyze the fundamental limits in providing deterministic performance guarantees to VBR video. Such a study has become feasible with a set of recently developed tight, i.e., necessary and sufficient, admission control tests for networks that employ Earliest-DeadlineFirst and Static Priority scheduling disciplines [9]. We present an optimal characterization of VBR video traffic for a deterministic service using what we term empirical envelopes. Empirical envelopes represent the most accurate, time-invariant, deterministic characterization of a traffic source. Using this optimal characterization, together with Earliest-Deadline-First packet scheduling and the exact admission control tests from [9], we provide insights into the highest achievable utilization for VBR video sources.

We also investigate the fundamental tradeoffs that must be considered for practical networking environments. For example, a realistic traffic characterization must be easily policeable by the network. Furthermore, the traffic characterization should be concise so that the traffic of each source can be described with a small number of parameters. We study the impact of selecting easily policeable and concise traffic characterizations on the utilization limits of the deterministic service. We also investigate the impact of selecting simpler packet schedulers, such as Static Priority and First-Come-First-Served, on the achievable network utilization. Finally, for the Static Priority packet scheduler we demonstrate the degree to which the accuracy of the admission control tests influences the utilization of a deterministic service.

The remainder of the paper is structured as follows. In $\S 2$, we describe the network components necessary to offer a service with deterministic delay bounds. In $\S 3$, we devise a method to generate an optimal traffic characterization, the empirical envelope, for VBR video sources. We present an algorithm that allows us to approximate the empirical envelope by an easily policeable deterministic traffic model. In $\S 4$, we use traces of MPEG-encoded VBR video traffic to empirically evaluate the limits of the deterministic approach as well as the abovementioned tradeoffs.

\section{Components of a Deterministic Network Service}

For a network to provide deterministic performance guarantees on throughput, delay, delay-jitter, and loss, it must be able to tightly control certain resources. The utilization of network resources are influenced by three components: (1) the traffic model used for the characterization of the worst-case traffic from a connection, (2) the scheduling discipline at the network multiplexers, and (3) the accuracy of the admission control tests. In this section, we review the tradeoffs involved in the selection of these components and their impact on a deterministic network service.

\subsection{Deterministic Traffic Models}

A traffic model for a deterministic service has several fundamental requirements. First, the model must be a worstcase characterization of the source to provide an absolute upper bound on a source's packet arrivals. Second, the model must be parameterized so that a source can efficiently specify its traffic characterization to the network. Next, the model should characterize the traffic as accurately as possible so that the admission control algorithms do not over-estimate the resources required by the connection. Finally, the model must be policeable so that the network can enforce a source's traffic characterization.

A worst-case representation of a traffic source may be described as follows. If the actual traffic of a connection is given by a function $A$ such that $A[\tau, \tau+t]$ denotes the traffic arrivals in the time interval $[\tau, \tau+t]$, an upper bound on $A$ can be given by a function $A^{*}$ if for all times $\tau \geq 0$ and all interval lengths $t \geq 0$ the following holds [1, 2]:

$$
A[\tau, \tau+t] \leq A^{*}(t)
$$

We refer to a function $A^{*}(t)$ that satisfies the property in (1) as a traffic constraint function. Note that a traffic constraint function provides a time-invariant bound on $A$, so that a source is bounded for every interval of length $t$.

In practice, a source specifies its traffic characterization with a parameterized model. The parameterized deterministic traffic model defines a traffic constraint function that bounds the source. For example, in the $\left(x_{\text {min }}, x_{\text {ave }}, I, s\right)$ model [4], $x_{\min }$ is the minimum packet inter-arrival time, $x_{\text {ave }}$ is the maximum average packet inter-arrival time over any time interval of length $I$, and $s$ is the maximum packet size. This worst-case parameterization of a source has an associated traffic constraint function given in Table 1. The $(\sigma, \rho)$ model [2] describes traffic in terms of a rate factor $\rho$ and a burstiness factor $\sigma$ such that during any time interval of length $t$, the traffic from a connection is less than $\sigma+\rho t$. In this study, we consider a $(\vec{\sigma}, \vec{\rho})$ model, which can be viewed as an extension to the $(\sigma, \rho)$ model. The $(\vec{\sigma}, \vec{\rho})$ model maintains a number $n$ of $(\sigma, \rho)$ pairs, where the amount of traffic in a time interval $t$ is restricted to $\min _{i}\left\{\sigma_{i}+\rho_{i} t\right\}$. A more general deterministic traffic model called the Deterministic Bounding Interval Dependent or $D$-BIND model [8] characterizes traffic by a family of rate-interval pairs $\left\{\left(R_{j}, I_{j}\right) \mid j=1,2, \cdots, n\right\}$. The D-BIND model defines an $n$ segment piece-wise linear traffic constraint function as given in Table 1 . The $(\vec{\sigma}, \vec{\rho})$ model may be viewed as a special case of the D-BIND model because it defines an $n$ segment piece-wise linear concave traffic constraint function.

Table 1 depicts the traffic constraint functions $A^{*}$ for the aforementioned deterministic traffic models. Since deterministic traffic models have associated traffic constraint functions, the admission control tests may be expressed in terms of $A^{*}$. A requirement on $A^{*}$ for the admission control tests is subadditivity, i.e., for all $t_{1} \geq 0$ and all $t_{2} \geq 0$ we have $A^{*}\left(t_{1}+t_{2}\right) \leq A^{*}\left(t_{1}\right)+A^{*}\left(t_{2}\right)[1,9]$.

Since the selection of the traffic model determines the shape of a traffic constraint function, from the perspective 


\begin{tabular}{|c|c|c|}
\hline Traffic Model & Traffic Constraint Function & \\
\hline$(\sigma, \rho)$ & $A^{*}(t)=\sigma+\rho t$ & \\
\hline$(\vec{\sigma}, \vec{\rho})$ & $A^{*}(t)=\min _{1 \leq i \leq n}\left\{\sigma_{i}+\rho_{i} t\right\}$ & \\
\hline$\left(x_{\text {min }}, x_{\text {ave }}, I, s\right)$ & $A^{*}(t)=\left\lfloor\frac{t}{I}\right\rfloor \cdot \frac{I \cdot s}{x_{a v e}}+\min \left\{\left\lceil\left(\frac{t}{I}-\left\lfloor\frac{t}{I}\right\rfloor\right) \cdot \frac{I}{x_{\min }}\right\rceil, \frac{I}{x_{\text {ave }}}\right\} \cdot s$ & \\
\hline D-BIND & $A^{*}(t)=\left\{\begin{array}{l}t R_{1} \\
\frac{I_{j-1} I_{j}\left(R_{j-1}-R_{j}\right)+t\left(R_{j} I_{j}-R_{j-1} I_{j-1}\right)}{I_{j}-I_{j-1}}\end{array}\right.$ & $\begin{array}{l}\text { for all } t \leq I_{1} \\
\text { for all } I_{j-1}<t \leq I_{j}\end{array}$ \\
\hline
\end{tabular}

Table 1: Traffic Models and Traffic Constraint Functions.

of achievable network utilization, the model should have a traffic constraint function that is as tight as possible so that the admission control algorithms do not over-estimate the resources required by the connection. While in general, a model with more parameters can achieve a more accurate traffic constraint function, the additional parameterization causes an increase in the complexity of policing the traffic model. Thus, the selection of an appropriate traffic model for a deterministic service must find a compromise between the high accuracy preferred by the admission control tests and the simplicity required for the implementation of traffic policers. The policing mechanisms must verify in real-time whether the traffic transmitted on an established connection adheres to a specified set of parameters of a deterministic traffic model. To ensure that the policing mechanisms can monitor and control traffic at high data rates, the complexity of the traffic model is limited. In [2], it was shown that a traffic model with a piece-wise linear concave traffic constraint function can be policed by a fixed number of leaky buckets. Since a leaky bucket can be implemented with a counter and a single timer [15], concavity of the $(\vec{\sigma}, \vec{\rho})$ model's traffic constraint functions ensures a simple implementation of the traffic policer.

\subsection{Packet Scheduling}

In a connection-oriented packet-switched network, packets from a particular connection traverse the network on a fixed path of switches and links. Each switch has a packet scheduler for each outgoing link. Since the packet scheduler can transmit only one packet at a time, it maintains a queue containing all packets waiting for transmission. Here we consider the following well-known scheduling disciplines at a packet scheduler for a set $\mathcal{N}$ of connections: First-ComeFirst-Served (FCFS), Static Priority (SP), and EarliestDeadline-First (EDF). Each of these disciplines has been investigated for use in bounded delay services [2, 4, 9, 16].

First-Come-First-Served schedulers transmit all packets in the order of their arrival. Since the maximum delay in a FCFS scheduler is the same for all connections $j \in \mathcal{N}$, all connections have an identical delay bound $d$.

In a Static Priority packet scheduler such as in [16], each connection $j \in \mathcal{N}$ is assigned a priority $p$ with $1 \leq p \leq P$, where a lower index indicates a higher priority. $\mathcal{C}_{p}$ is the set of connections with priority $p$, and all connections in $\mathcal{C}_{p}$ have the same delay bound $d_{p}$, with $d_{p}<d_{q}$ for $p<q$.
SP schedulers maintain one FCFS queue for each priority level, always selecting the first packet in the highestpriority FCFS queue for transmission.

With Earliest-Deadline-First scheduling [4], each connection $j \in \mathcal{N}$ is assigned a delay bound $d_{j}$, where the delay bound may be different for each connection. An EDF scheduler selects packets for transmission in increasing order of packet deadlines, where packet deadlines are calculated as the sum of the arrival time and the delay bound of a packet.

The selection of a particular scheduling discipline for a packet scheduler involves a tradeoff between the need to support a large number of connections with diverse delay requirements and the need for simplicity in the scheduling operations. For example, while a FCFS scheduler can be easily implemented, it can effectively support only one delay bound for all connections. On the other extreme, while an EDF scheduler can support a different delay bound for each connection, the scheduling operations of EDF are complex since they involve a search operation for the packet with the shortest deadline. EDF scheduling is shown to be optimal in [9] in the sense that if any packet scheduling method can meet a set of connections' delay constraints, so can EDF.

\subsection{Admission Control Tests}

While the number of admission control tests can be large, including tests for availability of transmission capacity, CPU power, buffer space, etc., the most crucial admission test in a network with a deterministic service is the delay bound test. The delay bound test verifies that, for all connections, the delay of each packet is less than its required delay bound. Most other admission control tests, including those that verify throughput and delay-jitter guarantees, can be directly derived from the delay bound test.

Note that there is a tradeoff between the accuracy and the complexity of admission control tests. A less accurate admission control algorithm may be less computationally expensive, however, it may reject a connection even though the acceptance of the connection will not affect the QoS, therefore result in a lower utilization of the network.

Tables 2 and 3 present conditions that must be satisfied to pass delay bound tests for FCFS, SP, and EDF packet schedulers. In the tables, the link speed is normalized to 1 , $s_{k}$ denotes the maximum packet size for connection $k$, and 


\begin{tabular}{|l|lr|}
\hline Delay Bound Test & Condition \\
\hline \hline FCFS Exact & $d \geq \sum_{j \in \mathcal{N}} A_{j}^{*}(t)-t+\max _{k \in \mathcal{N}} s_{k}$ & for all $t \geq 0$. \\
\hline \hline SP Exact & $\exists \tau \leq d_{p}-s_{p}^{\text {min }}$ such that: \\
& $t+\tau \geq \sum_{j \in \mathcal{C}_{p}} A_{j}^{*}(t)+\sum_{q=1}^{p-1} \sum_{j \in \mathcal{C}_{q}} A_{j}^{*}(t+\tau)-s_{p}^{\text {min }}+\max _{r>p} s_{r} \quad$ for all $p, t \geq 0$. \\
\hline \hline EDF Exact & $t \geq \sum_{j \in \mathcal{N}} A_{j}^{*}\left(t-d_{j}\right)+\max _{d_{k}>t} s_{k}$ & for all $t \geq d_{1}$. \\
\hline
\end{tabular}

Table 2: Exact delay bound tests for FCFS, SP, and EDF packet schedulers.

\begin{tabular}{|l|ll|}
\hline Delay Bound Test & Condition \\
\hline \hline SP Sufficient 1 & $t \geq \sum_{j \in \mathcal{C}_{p}} A_{j}^{*}\left(t-d_{p}\right)+\sum_{q=1}^{p-1} \sum_{j \in \mathcal{C}_{q}} A_{j}^{*}(t)+\max _{r>p} s_{r}$ & for all $p, t \geq d_{p}$. \\
\hline \hline SP Sufficient 2 & $d_{p} \geq \sum_{q=1}^{p} \sum_{j \in \mathcal{C}_{q}} A_{j}^{*}\left(d_{p}\right)+\max _{r>p} s_{r}$ & for all $p$. \\
\hline
\end{tabular}

Table 3: Sufficient delay bound tests for an SP packet scheduler.

$s_{p}^{\text {min }}$ denotes the minimum packet size for any connection with priority $p$. Table 2 shows the necessary and sufficient delay bound tests ( Exact Tests") for each scheduler. Formal derivations of these tests can be found in [2, 9]. Since the exact admission test for an SP scheduler is computationally very complex, Table 3 presents less accurate tests ( "Sufficient Tests") that require less computation [9, 17]. Note that each test is formulated in terms of $A^{*}$ so that it can be applied to the traffic models discussed in $\$ 2.1$.

As an example, we present an informal derivation of the delay bound test for the FCFS packet scheduler. Recall that the FCFS packet scheduler can offer only one delay bound $d$ to all connections. Thus, a delay bound test for FCFS simply verifies that the maximum waiting time of any packet never exceeds $d$. If the scheduler is never idle in the time interval $[0, t]$, then the maximum waiting time of a packet that arrives at time $t$ is determined by the maximum backlog in the transmission queue $\left(\sum_{j \in \mathcal{N}} A_{j}[0, t]-t\right)$ and the largest remaining transmission time of any packet that is in transmission at time $t\left(\max _{k \in \mathcal{N}} s_{k}\right)$. Since $A_{j}[0, t] \leq$ $A_{j}^{*}(t)$ by Equation (1), we are guaranteed not to have a deadline violation at time $t+d$ if and only if the following holds at time $t: d \geq \sum_{j \in \mathcal{N}} A_{j}^{*}(t)-t+\max _{k \in \mathcal{N}} s_{k}$. Applying the argument to all times $t \geq 0$ yields the delay bound test given in Table 2. As alluded to above, other admission control tests can be directly derived from this bound on queueing delay $d$. For example, if the buffer space that is available for each connection connection $j$ is at least $A_{j}^{*}(d)$, then packets will never be dropped due to buffer overflows.

The above delay bound at a single server can be extended across multiple servers to obtain an end-to-end delay bound. Depending on the class of service disciplines considered, various techniques may be applied. Several examples are as follows. First, end-to-end delay bounds for the class of work-conserving service disciplines are de- rived in [3]. This is achieved by calculating a bound on the transformation of a stream's constraint function $A^{*}(t)$ as it traverses each consecutive hop. An end-to-end delay bound can then be calculated by summing the local, per-hop delay bounds. Second, for a class of service disciplines called rate-controlled service disciplines [18], e.g., Rate-Controlled Static Priority [16] and a rate-controlled variation of EDF [4], a stream's original constraint function $A^{*}(t)$ is restored at each hop along the path via a rate controller. This prevents the traffic constraint function from becoming "burstier" as the stream traverses multiple hops, as occurs in [3]. Finally, [11] utilizes properties specific to the Generalized Processor Sharing (GPS) service discipline, which allows the derivation of tight end-to-end delay bounds for networks of GPS servers with leaky bucket constrained sources.

\section{Deterministic Characterizations of VBR Video Traffic}

Here we present the optimal characterization of VBR video traffic for a deterministic service using empirical envelopes. We then show how traffic models such as the $(\vec{\sigma}, \vec{\rho})$ model can be viewed in terms of the empirical envelope and we present an algorithm for deriving the $(\vec{\sigma}, \vec{\rho})$ parameters from the envelope.

In this paper, we use traces of video compressed with the MPEG compression algorithm as examples of VBR video traffic. Figure 1 shows a typical segment of a trace of MPEG compressed video obtained from a recorded sequence of advertisements. An MPEG coder generates three types of frames: I frames that use intraframe compression, and $P$ and $B$ frames that are transmitted between $I$ frames that use interframe compression. While $P$ frames 
(predicted frames) are coded based on only past frames, $B$ frames (bidirectional frames) are coded based on both a past and a future reference frame. Thus, $I$ frames exploit spatial locality, while $P$ and $B$ frames exploit temporal locality. More details of the MPEG algorithm may be found in [6]. The behavior of the coder is clearly visible from the dynamics of the trace. Specifically, the frame pattern (in this case $I B B P B B$ ) can be seen since the $I$ frames tend to be the largest, $B$ the smallest, and $P$ in between.

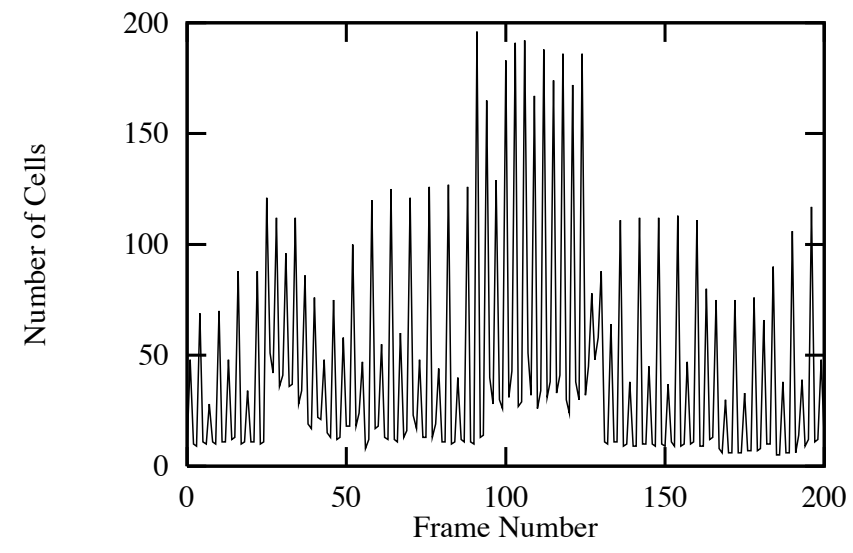

Figure 1: Traffic of an MPEG video stream.

\subsection{The Empirical Envelope}

As described in $§ 2.1$, each deterministic traffic model has an associated traffic constraint function $A^{*}(t)$ that provides an upper bound on the source's arrivals in any interval of length $t$. The empirical envelope presented next provides the most accurate traffic constraint function for a given video trace which allows evaluation of the maximum achievable utilization for a deterministic service to VBR video. For any scheduling discipline, using the exact admission control tests with the empirical envelope results in the highest network utilization achievable in a deterministic service. Although the empirical envelope will be shown to lack important practical properties such as the ability to be efficiently specified or policed, it does provide an optimal benchmark for evaluating deterministic traffic models. Toward this end, the empirical envelope $E^{*}$ is defined as the most accurate traffic constraint function for an arrival function $A$ as [1]:

$$
E^{*}(t)=\max _{\tau>0} A[\tau, \tau+t] \quad \forall t>0
$$

Equation (2) indicates that $E^{*}(t)$ is a time-invariant, tight bound on arrivals in any interval of length $t$. Note that the tightness of $E^{*}$ implies that any traffic constraint function $A^{*}$ for an arbitrary traffic model satisfies $A^{*}(t) \geq E^{*}(t)$ for all $t$. Since the admission control tests in Tables 2 and 3 are based on the traffic constraint functions $A^{*}$, using the most accurate or minimum constraint function $E^{*}$ in these tests results in the highest achievable network utilization. That is, from the tests of Tables 2 and 3 , it follows that the tightest delay conditions are obtained if $A^{*}$ is selected such that $A^{*} \equiv E^{*}$. Thus, the empirical envelope can be used as a benchmark for comparing deterministic traffic models.

The empirical envelope for a given video trace may be obtained in the following manner. For the traces analyzed in this paper, the inter-frame time $T$ is fixed and each video frame is fragmented into 48 byte ATM cells which are transmitted at equally-spaced intervals over the frame time $T$. If the sequence of frame sizes of video source $j$ is given by $\left\{f_{1}, f_{2}, \ldots, f_{N}\right\}$, and if the transmission of this sequence starts at time 0 , the arrival function $A$ which describes the cumulative number of cell arrivals up to time $t$ is given as follows:

$$
A[0, t]=\sum_{i=1}^{\left\lfloor\frac{t}{T}\right\rfloor} f_{i}+\left(\frac{t}{T}-\left\lfloor\frac{t}{T}\right\rfloor\right) f_{\left\lceil\frac{t}{T}\right\rceil}, \quad 0 \leq t \leq N \cdot T
$$

The first term on the right-hand side of equation (3) denotes the number of cells from frames that are fully transmitted at time $t$, and the second term gives the number of transmitted cells from the frame that is being transmitted at time $t$. The empirical envelope may then be constructed by combining Equations (2) and (3).

Alternatively, $E^{*}$ can be obtained directly from the sequence of frames $\left\{f_{1}, f_{2}, \ldots, f_{N}\right\}$ by first calculating:

$$
E^{*}(i \cdot T)=\max _{0<k<N-i} \sum_{j=k}^{k+i} f_{j} \quad \text { for } i=1,2, \ldots, N
$$

The values of the empirical envelope at times that are not multiples of the frame time are then obtained by spacing the cells or packets in $E^{*}((i+1) \cdot T)-E^{*}(i \cdot T)$ evenly over the frame time $[i \cdot T,(i+1) \cdot T]$. Figure 2 illustrates the arrival function $A$ and the empirical envelope $E^{*}$ for the MPEG video trace shown in Figure 1.

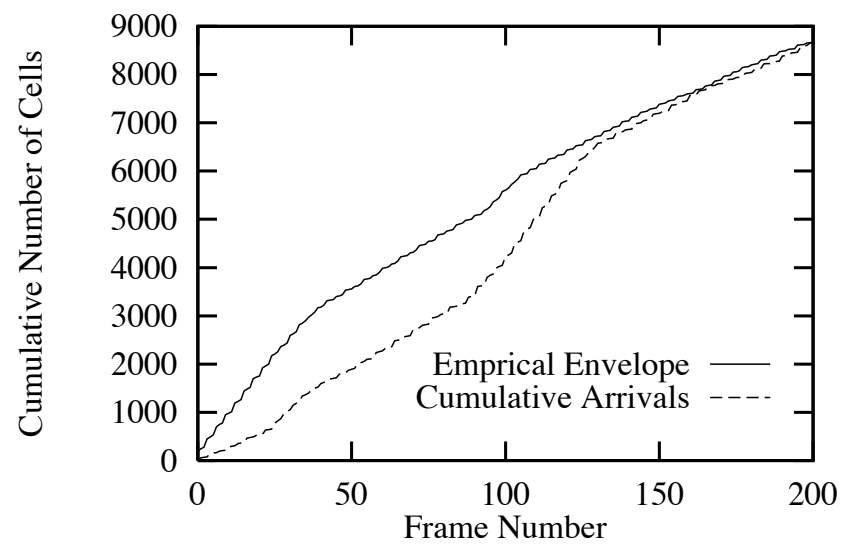

Figure 2: The accumulated traffic $A[0, t]$ of the MPEG stream from Figure 1 and its empirical envelope $E^{*}(t)$.

\subsection{Obtaining Model Parameters from the Empirical Envelope}

Here we present an algorithm that uses the empirical envelope to construct a traffic constraint function $A^{*}(t)$ that 
conforms to the $(\vec{\sigma}, \vec{\rho})$ traffic model. Recall from $\S 2.1$ that the $(\vec{\sigma}, \vec{\rho})$ traffic model is defined by a set of parameter pairs $\left\{\left(\sigma_{i}, \rho_{i}\right) \mid i=1,2, \ldots n\right\}$ with a traffic constraint function given by $A^{*}(t)=\min _{i}\left\{\sigma_{i}+\rho_{i} t\right\}$. Note that this traffic constraint function $A^{*}(t)$ is guaranteed to be concave and can thus be policed with an $n$-level leaky bucket $[2,8]$. The algorithm, shown in Figure 3, assumes that the values of the empirical envelope are available up to some time limit $\tau$. It determines the number of $\left(\sigma_{i}, \rho_{i}\right)$ pairs that are needed to approximate the envelope and selects the parameter values for each $\left(\sigma_{i}, \rho_{i}\right)$ pair.

The parameter selection by the algorithm proceeds as follows. For the given time $\tau$, a parameter pair $\left(\sigma_{i}, \rho_{i}\right)$ and a new time value $0 \leq \tau^{\prime}<\tau$ are selected such that $E^{*}(\tau)=\sigma_{i}+\rho_{i} \tau, E^{*}(t) \leq \sigma_{i}+\rho_{i} t$ for all $0 \leq t \leq \tau$, and $E^{*}\left(\tau^{\prime}\right)=\sigma_{i}+\rho_{i} \tau^{\prime}$. This procedure is repeated with a newly calculated time value $\tau^{\prime}$ as long as $\tau^{\prime}$ is positive. From Equation (2) and the discussions above, it can be seen that the algorithm constructs $A^{*}$ that is a piece-wise linear concave upper approximation to $E^{*}$.

\begin{tabular}{|c|c|}
\hline Input: An empirical envelope $E^{*}$ and a time $\tau$. \\
Output: A set of leaky bucket parameters. \\
\hline 1. & Procedure Find_Parameters $\left(E^{*}, \tau\right)$ \\
2. & $n=0$ \\
3. & While $\tau>0$ Do \\
4. & $n=n+1$ \\
5. & $\sigma_{n}=\max _{0 \leq t<\tau}\left\{\frac{1}{\tau-t}\left(\tau E^{*}(t)-t E^{*}(\tau)\right)\right\}$ \\
6. & $\rho_{n}=\frac{E^{*}(\tau)-\sigma_{n}}{\tau}$ \\
7. & Output $\left(\sigma_{n}, \rho_{n}\right)$ \\
8. & $\tau=\min \left\{t \mid \sigma_{n}+\rho_{n} t=E^{*}(t)\right\}$ \\
9. & End While \\
10. & End Procedure \\
\hline
\end{tabular}

Figure 3: An algorithm to determine $(\vec{\sigma}, \vec{\rho})$ parameters.

Figure 4 illustrates the operations of the algorithm with a simple example that results in the calculation of two $\left(\sigma_{i}, \rho_{i}\right)$ pairs. Figure 4(a) depicts the selection of parameters for the pair $\left(\sigma_{1}, \rho_{1}\right)$ as calculated in the first iteration of the algorithm with the given empirical envelope $E^{*}$ and interval $\left[0, t_{1}\right]$. The parameters $\sigma_{1}$ and $\rho_{1}$ and the new time value $t_{2}$ are chosen such that the curve $\sigma_{1}+\rho_{1} t$ is never below the empirical envelope and is identical to the envelope at times $t_{1}$ and $t_{2}$. Also shown in Figure 4(a) is the selection of time $t_{2}$ which determines the starting time for the next iteration of the algorithm.

Figure 4(b) illustrates the determination of the second pair $\left(\sigma_{2}, \rho_{2}\right)$ which is done analogously to the selection of $\left(\sigma_{1}, \rho_{1}\right)$ in Figure 4(a). The algorithm terminates after the calculation of the second pair of parameters since $t_{3}=0$. The final result is shown in Figure 4(c) where we show the traffic constraint function produced by the algorithm as a bold curve. In this example, two parameter pairs $\left(\sigma_{1}, \rho_{1}\right)$ and $\left(\sigma_{2}, \rho_{2}\right)$ determine the traffic constraint function, $A^{*}(t)=\min \left\{\sigma_{1}+\rho_{1} t, \sigma_{2}+\rho_{2} t\right\}$.

Finally, as an illustrative example to emphasize the potential simplicity of approximating $E^{*}$ for VBR video, we note as in [8] that for MPEG video sources, one possible concise and simple-to-obtain parameterization of $A^{*}$ is to use knowledge of the frame pattern along with a parameterization of the largest sized $I$ frame, $B$ frame, and $P$ frame. With this alternative "worst-case" characterization, a pessimistic approximation to $E^{*}$ is easily obtained by constructing $A^{*}$ as a transmission of the largest $I$ frame, followed by 2 transmissions of the largest $B$ frame and so on. In essence, any $A^{*}$ that is a subadditive upper approximation to $E^{*}$ can be used as a traffic constraint function, while, as described in $\$ 2.1$, the shape of $A^{*}$ affects its policeability, and, as explored in the next section, the tightness of $A^{*}$ affects the achievable network utilization.

\section{Evaluation of Design Choices for Deterministic Services}

In this section, we use several traces of MPEG-compressed video to empirically evaluate the fundamental limits and tradeoffs of deterministic services as described in the previous sections. First, the theoretical limits on the efficiency of a deterministic network service are illustrated by combining the tightest traffic constraint function $E^{*}$, with an exact admission control test for the best packet scheduler, EDF. With these utilization limits as a benchmark, we explore the achievable utilization of networks that use more practical traffic models, packet schedulers, and admission control tests. In all experiments, we consider a single multiplexer that operates at $45 \mathrm{Mbps}$, corresponding to the transmission rate of a T3 line. As described in Section 2.3, end-to-end delay bounds may be calculated as an accumulation of the local delay bounds investigated below. While there is not a consensus on the exact range of acceptable delay bounds for interactive applications, some discussion of this is found in [12].

In our experiments, we use traces of two MPEG-1 compressed video sequences that were encoded with constant quality using the Berkeley MPEG-encoder [13]. The first trace is a 30-minute sequence taken from the entertainment film "Jurassic Park" which we refer to as Movie. Movie is digitized to 384 by 288 pixels and compressed at 24 frames per second with frame pattern $I B B P B B P B B P B B$. The second trace, referred to as Lecture, is a 10-minute sequence showing a videotaped lecture where the camera zooms in and out, and pans between the speaker and her transparencies. The Lecture sequence is digitized to 160 by 120 pixels and compressed at 30 frames per second using the frame pattern $I B B P B B$. These video sequences were selected because they have very different characteristics in terms of resolution, frame pattern, and frequency of scene changes.

\subsection{Maximum Achievable Utilization of a Deterministic Service}

In the first experiment, we show the maximum link utilization that can be achieved for a deterministic service using the exact admission control test for an EDF scheduler from Table 2 and empirical envelopes $E^{*}$ as traffic constraint functions. Note that this scenario corresponds 


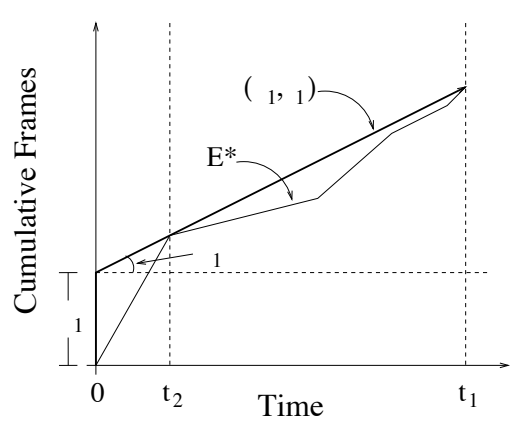

(a) Selection of $\sigma_{1}, \rho_{1}$, and $t_{2}$.

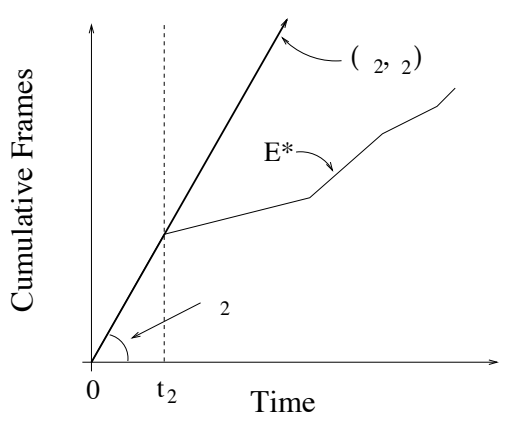

(b) Selection of $\sigma_{2}$ and $\rho_{2}$.

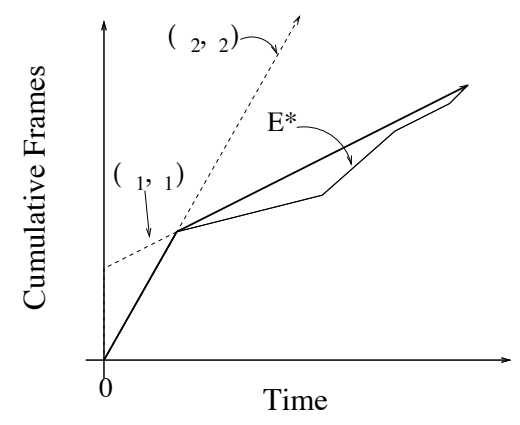

(c) Traffic constraint function.

Figure 4: Parameter selection by the algorithm in Figure 3.

to using the tightest traffic constraint function in combination with the most accurate admission control test for the best packet scheduler. Here we consider the case of homogeneous sources with identical delay requirements. ${ }^{2}$ Heterogeneous sources (combinations of the Movie and Lecture sequences) with heterogeneous delay bounds are considered in $\$ 4.3-4.5$.

Figures 5(a) and 5(b) illustrate the maximum number of Lecture and Movie connections, respectively, that can be admitted for a deterministic service. In each graph, the number of connections that can be simultaneously supported is plotted as a function of the delay bound of those connections. Both figures also include two reference cases, referred to as Peak Rate and Average Rate.

- The Peak Rate plot shows the maximum number of connections that can be accepted if admission control tests are based on peak rate allocation. The peak rate is assumed to be the ratio of the largest video frame and the inter-frame time. More formally, the peak rate $\rho_{\text {peak }}$ of a sequence of frames with sizes $\left\{f_{1}, f_{2}, \ldots, f_{N}\right\}$ and with fixed inter-frame time $T$ is given by:

$$
\rho_{\text {peak }}=\frac{\max _{0<j \leq N} f_{j}}{T}
$$

- The Average Rate of a video sequence is determined by the average traffic rate over the length of the video sequence. Denoting the average rate by $\rho_{a v g}$ we obtain:

$$
\rho_{a v g}=\frac{\sum_{j=1}^{N} f_{j}}{N \cdot T}
$$

If the video sequences are repeated indefinitely, an allocation based on average rates ensures that all packet delays are finite.

The general trend of these curves is that as the delay bound increases, more connections can be established. One important observation is that a reasonable network utilization for deterministic service can be achieved even when the delay bound is fairly small. In the case of Lecture (Figure $5(\mathrm{a})$ ), an average utilization of $55.8 \%$ can be achieved with a delay bound of $50 \mathrm{msec}$. Note that these utilizations are considerably higher than the $22.5 \%$ utilization achieved

\footnotetext{
${ }^{2}$ Note that EDF, SP, and FCFS produce the same schedule when all connections have the same local delay bound
}

with a peak rate allocation scheme. Recall from Subsection $\$ 2.3$ that we can bound the maximum buffer requirements of a connection with delay bound $d$ and traffic constraint function $A^{*}$ by $A^{*}(d)$. With this bound, the per-stream buffer requirements of the lecture sequence with a delay bound of 50 msec are given by 7.0 kilobytes per connection. This corresponds to 146 cells per connection or 9,782 cells for all 67 connections.

Figure 5(b) shows that the achievable utilization for a given delay bound is considerably lower for the Movie sequence than for the Lecture sequence. Since both sequences are encoded with the same software tool [13], the difference in utilization results from the different frame pattern used for the Movie sequence and its additional motion and scene changes. Regardless, admission control tests that use the empirical envelope still provide an improvement over the $10.5 \%$ utilization achievable with admission control tests that use a peak-rate allocation scheme.

\subsection{Tradeoffs in Number of Traffic Param- eters}

Here we explore the utilization tradeoffs in a deterministic service when using a practical deterministic traffic model rather than the empirical envelope. With the exact admission control test for an EDF scheduler, we use the $(\vec{\sigma}, \vec{\rho})$ model to characterize the traffic and the empirical envelope $E^{*}$ as a benchmark to compare the utilization achieved for various numbers of $\left(\sigma_{i}, \rho_{i}\right)$ pairs.

Figures $6(\mathrm{a})$ and $6(\mathrm{~b})$ depict the respective number of homogeneous Lecture and Movie connections that can be supported as a function of delay bound for various numbers of $\left(\sigma_{i}, \rho_{i}\right)$ pairs. When a number $j$ of $\left(\sigma_{i}, \rho_{i}\right)$ pairs is specified, the $j$ pairs used are the those with the smallest values of $\sigma_{i}$, e.g., the single pair $\left(\sigma_{1}, \rho_{1}\right)$ would be the one with the smallest value of $\sigma_{i}$.

The first observation from Figure 6 is that as more $\left(\sigma_{i}, \rho_{i}\right)$ pairs are used, more connections can be accepted. The reason for this is that a traffic constraint function $A^{*}$ defined with more parameters can better approximate $E^{*}$, allowing the acceptance of more connections. However, it should also be noted that the benefit of adding additional $\left(\sigma_{i}, \rho_{i}\right)$ pairs decays quickly. Figure $6(\mathrm{a})$ shows that a characterization of Lecture that uses two $\left(\sigma_{i}, \rho_{i}\right)$ pairs results in the 


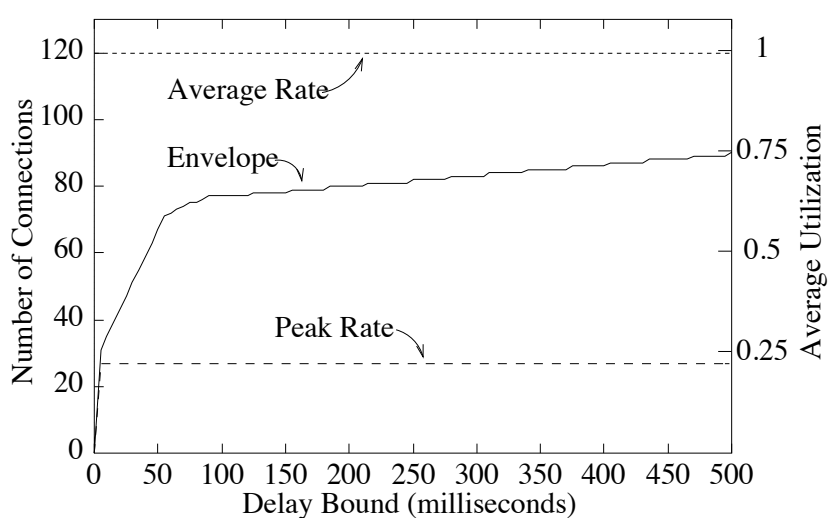

(a) Lecture

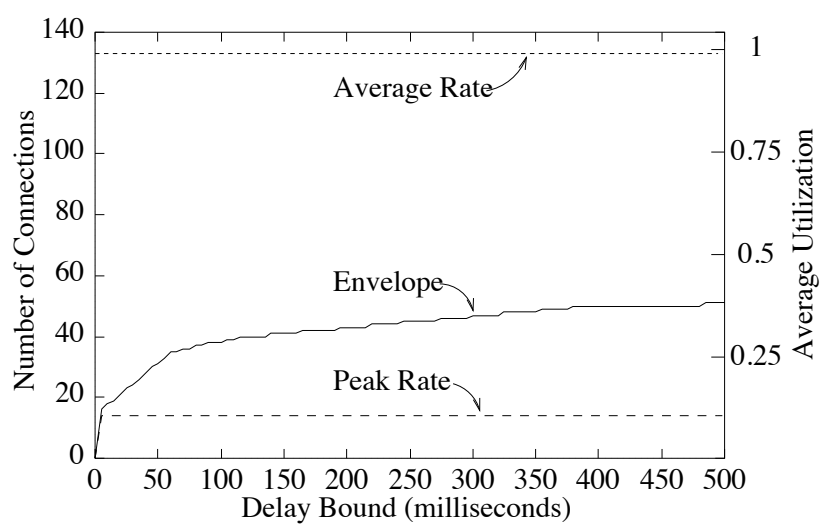

(b) Movie

Figure 5: Fundamental limits of a deterministic network service.

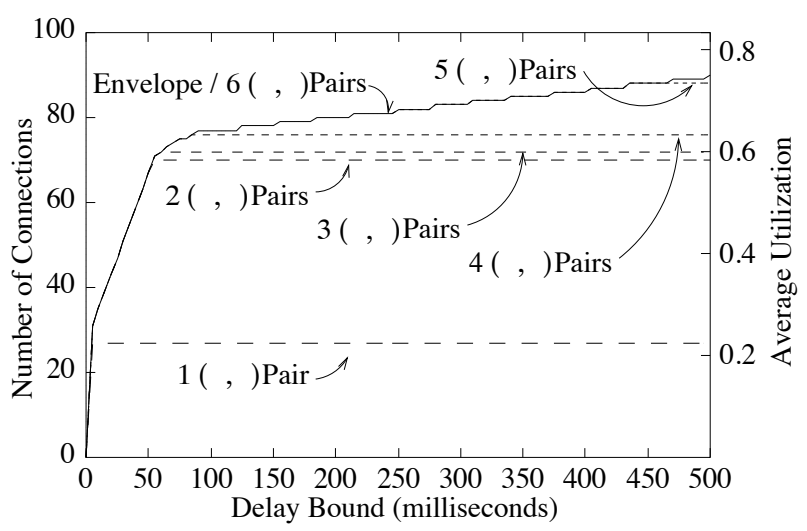

(a) Lecture

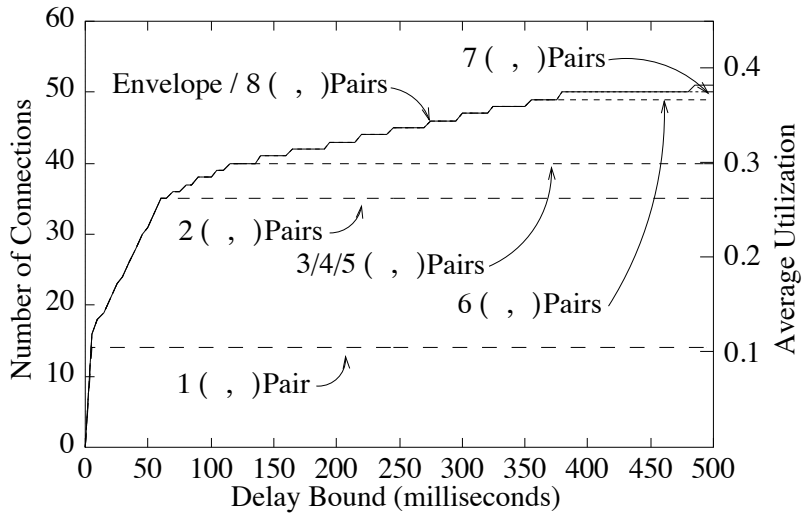

(b) Movie

Figure 6: A comparison of different traffic constraint functions.

same network utilization as the empirical envelope function when the delay bound is less than 55 msec. With six pairs, the same network utilization as for the empirical envelope can be achieved for almost all values of the delay bound, i.e., a seventh pair introduces no additional benefit. Since the $(\vec{\sigma}, \vec{\rho})$ model's $A^{*}$ forms a concave piece-wise linear upper bound of $E^{*}$, one may not expect that it can ever achieve the same utilization as the empirical envelope. The reason that it does in this case is that the advantage of considering a non-concave constraint function is only apparent with heterogeneous sources. Further discussion of this concavity issue may be found in [8], which allows a non-concave constraint function with the D-BIND model.

\subsection{Tradeoffs in Packet Scheduling}

In this section, we compare the performance of the three different packet schedulers discussed in $\$ 2.2$, namely EDF, $\mathrm{SP}$ and FCFS. We use the exact admission control tests from Table 2 with empirical envelopes as traffic constraint functions, and consider two types of connections: those carrying the Movie sequence and those carrying the Lecture sequence. All connections of the same type have identical delay requirements.

For each of these schedulers, Figure 7 shows the maximum number of Lecture and Movie connections that can be simultaneously supported by the network. Each curve in the figures corresponds to a pair of delay bounds for the Lecture and Movie connection types. Thus, the relative performance of the scheduling disciplines can be evaluated by comparing the contours of each plot. For reference purposes, we include the peak rate allocation curve.

By comparing Figure 7(a) and (b), it can be seen that the contour plots are similar. Thus, for our experiment that requires only a limited number of different delay bounds, an SP scheduler can support connections almost as well as an EDF scheduler. Alternatively, Figure 7(c) shows the advantages of EDF and SP disciplines over the FCFS discipline: since FCFS disciplines can, in effect, guarantee only a single delay bound, the number of Movie connections is limited by the tighter delay bound of the Lecture traffic.

\subsection{Tradeoffs in Admission Tests}

This experiment illustrates the impact of the accuracy of admission control tests on the utilization limits for the SP scheduler. Using the empirical envelope $E^{*}$ for traffic constraint functions, we consider the following admission control tests for the SP scheduler: "SP Exact" from Table 2, and "SP Sufficient I" and "SP Sufficient 2" from Table 3. These admission control tests vary in their computational complexity. If there are $P$ priority levels and the constraint 


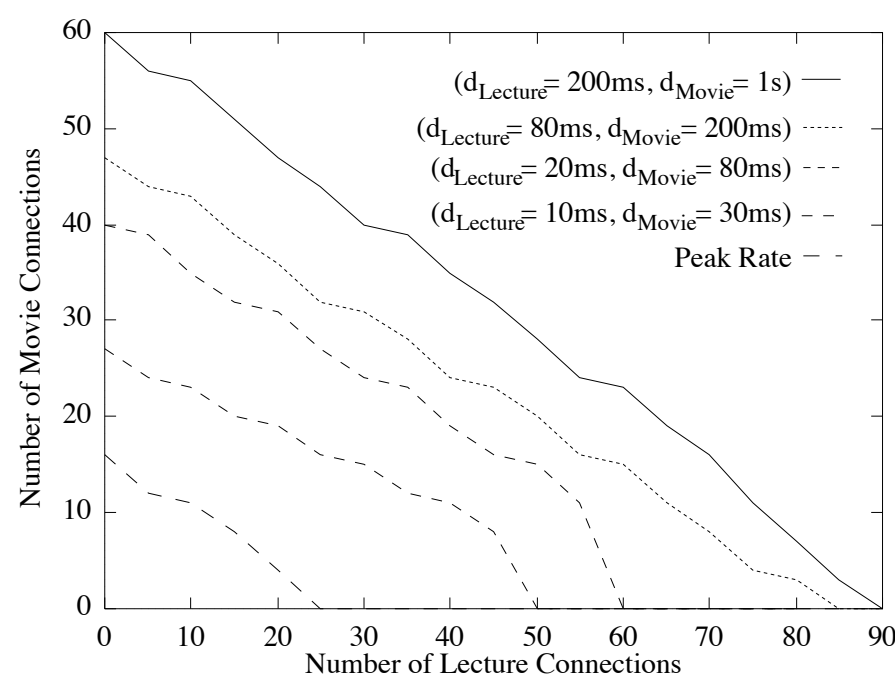

(a) EDF

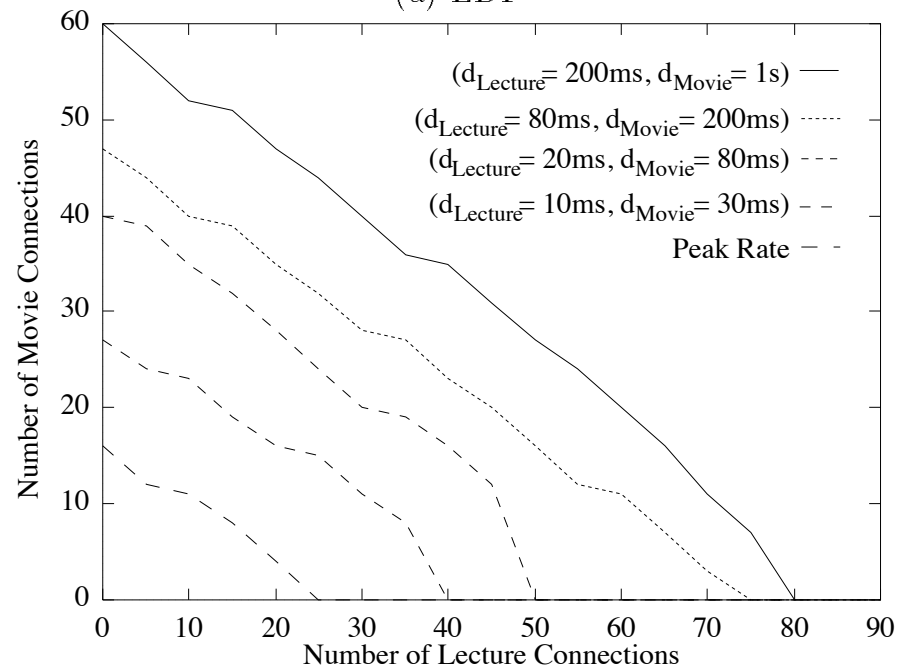

(b) $\mathrm{SP}$

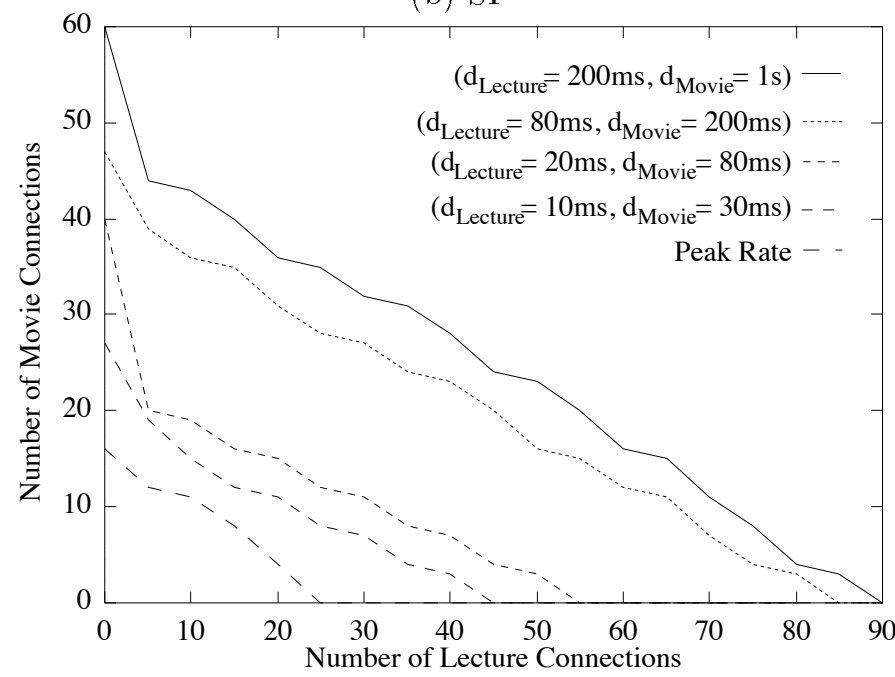

(c) FCFS

Figure 7: A comparison of packet schedulers. function is $E^{*}$ as derived from a frame sequence of length $N$, then the computational complexities of the admission control tests are as follows: the test SP Exact has complexity $O\left(P N d_{P}\right)$; the test $S P$ Sufficient 1 has complexity $O(P N)$; and the test $S P$ Sufficient 2 has complexity $O(P)$. If the constraint function is based on a parameterized traffic model rather than $E^{*}$, these complexities may be considerably reduced [9].

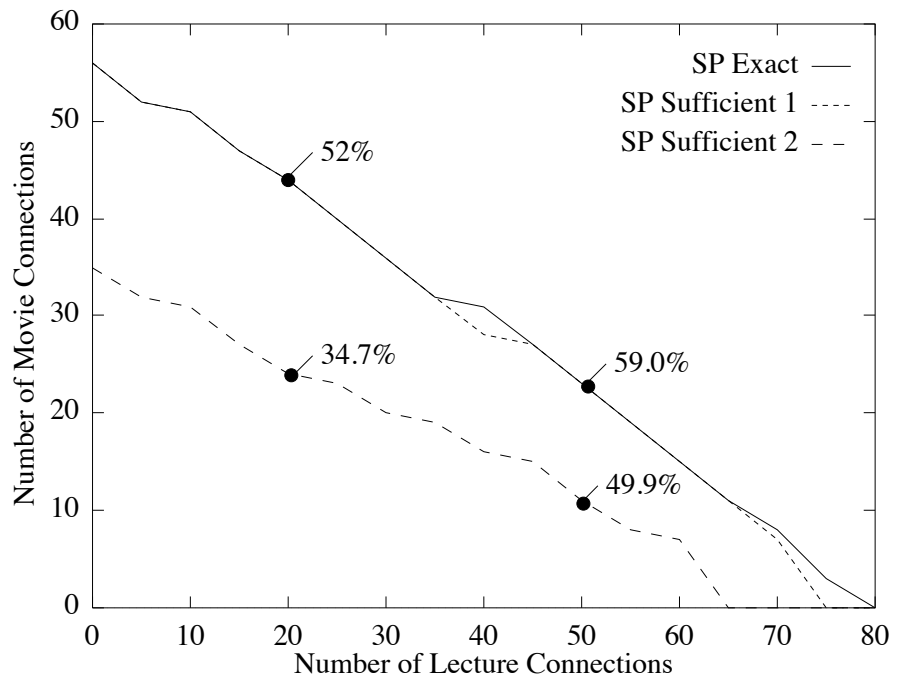

Figure 8: A comparison of admission control tests.

Figure 8 depicts the number of Lecture and Movie connections that can be simultaneously supported for each of the three admission control tests for the case in which $d_{\mathrm{Movie}}=150 \mathrm{msec}$ and $d_{\text {Lect }}=500 \mathrm{msec}$. For reference purposes, we depict several average utilization values expressed in terms of percentage of capacity. In this experiment, the admission control test SP Sufficient 1 achieves a utilization that is almost as high as the slightly more complex admission control test SP Exact. However, with a simpler admission control test such as SP Sufficient 2, the achievable utilization is substantially lower. Since the admission test $S P$ Sufficient 1 achieves a utilization very close to that of $S P$ Exact, it may be worthwhile to explore sufficient conditions for admissions tests.

\subsection{Summary of Tradeoffs}

In the previous three experiments, we analyzed the tradeoffs involved in selecting network components in isolation. Here we evaluate the utilization of a system that simultaneously considers choices for the traffic constraint functions, packet schedulers, and admission control tests. In particular, we select a Tradeoff case, which uses SP scheduling, the SP Sufficient 1 admission control tests from Table 3, and a traffic characterization consisting of $3(\sigma, \rho)$ pairs.

The Tradeoff case is compared to two reference cases. The first reference case, referred to as Best-Possible, is an upper bound and represents the case of EDF scheduling, exact admission control tests, and the empirical envelope for the source characterization. The second reference case, 
referred to as Peak Rate, is based on the peak rate allocation as used in the previous examples.

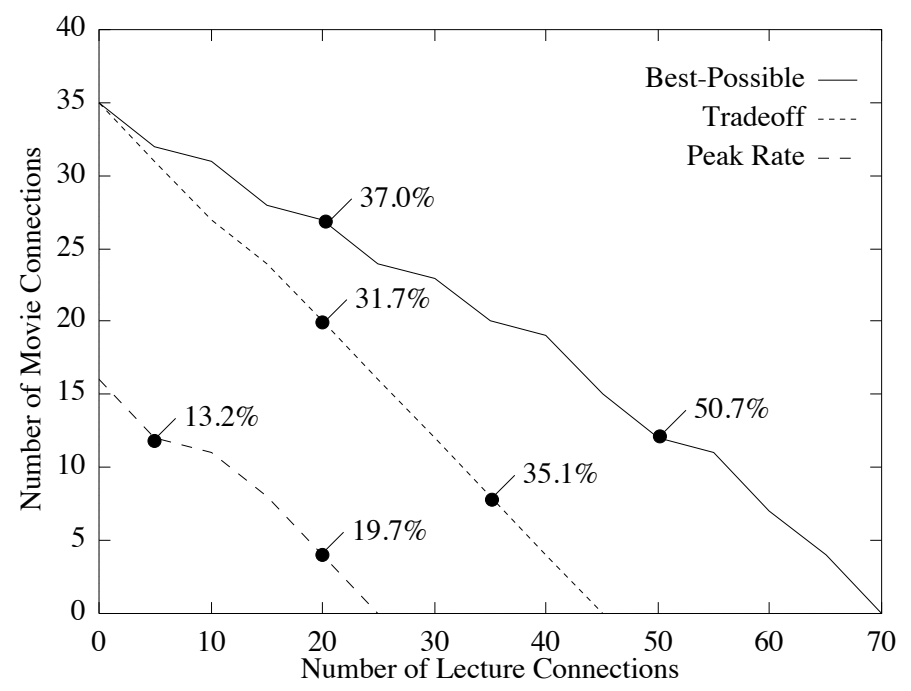

Figure 9: Achievable utilization with all tradeoffs.

Figure 9 depicts the maximum number of admissible connections for the three cases. For all cases, we set the delay bound for the Lecture connections to $30 \mathrm{msec}$ and the delay bound of the Movie connections to $50 \mathrm{msec}$.

The figure demonstrates that reasonable network utilizations of $25 \%$ to $40 \%$ can be achieved for deterministic service to VBR video even when taking into account practical considerations such as the need for simple schedulers, policing mechanisms, traffic models, and admission control tests. Moreover, these utilizations are considerably above the $16 \%$ utilization that is on the average achievable with a peak rate allocation scheme.

\section{Conclusions}

Providing deterministic guarantees requires the network to allocate resources according to the worst-case scenario. A common belief is that this requires a peak-rate resource allocation scheme that would significantly under-utilize the network when sources have high peak-to-average-rate ratios. However, previous work has shown that a deterministic service does not require a peak-rate allocation scheme even when deterministic performance guarantees are provided to bursty traffic $[9,17]$. Yet, to assess the applicability of the deterministic approach to VBR video, several important questions must be answered. For example, how high is the achievable utilization for real VBR video traffic when deterministic services are provided? What are the factors that affect the network utilization when deterministic services are provided? What are the tradeoffs between the achievable network utilization and other network design goals?

In order to address the above issues, we have studied the fundamental limits in achievable network utilization for deterministic service. We identified the main factors that influence the utilization of a network with deterministic services as (1) the deterministic traffic model used to characterize the source traffic, (2) the packet scheduling disciplines at the network switches, and (3) the accuracy of the admission control tests. The highest possible utilization is achieved for deterministic service in the "best possible" case with (1) empirical envelopes to characterize sources, (2) EDF scheduling disciplines at the network multiplexers, and (3) necessary and sufficient conditions for admission control tests. We quantified the limits of a deterministic service to VBR video by using traces of MPEG compressed video in the scenario above and found that considerable gains over a peak rate allocation scheme are possible.

Because this best possible scenario has limitations in its applicability to practical networks, we investigated fundamental tradeoffs for deterministic service. These tradeoffs include (1) considering a simple-to-police $n$ level leaky bucket $(\vec{\sigma}, \vec{\rho})$ traffic model with various values of $n$, (2) considering scheduling disciplines such as Static Priority and FCFS that do not require the sorted priority queue that EDF requires, and (3) considering fast admission control tests that employ sufficient schedulibility conditions rather than necessary and sufficient. Using the MPEG traces, we have shown the degree to which these factors impact the efficiency of a deterministic service to VBR video. Our experiments indicate that even in environments that consider the above tradeoffs, achievable utilizations are considerably above those obtained with a peak rate allocation scheme with average utilizations typically in the range of $25 \%$ to $40 \%$ for multiplexer delay bounds in the range of 30 to 50 msec.

As a final note, although it is tempting to draw conclusions such as "a concave constraint function with several linear segments is accurate enough to characterize VBR video" or "SP schedulers perform almost as well as EDF", we would like to caution readers to notice that our experiments are based on only two video traces. Connections with more diverse traffic characteristics and performance requirements are needed to further explore these issues. We leave further investigation of this to future work.

\section{Acknowledgements}

The authors are grateful to Sandia National Laboratories and to the University of Wuerzburg for the traces of MPEG compressed video.

\section{References}

[1] C. Chang. Stability, queue length, and delay of deterministic and stochastic queueing networks. IEEE Transactions on Automatic Control, 39(5):913-931, May 1994.

[2] R. Cruz. A Calculus for Network Delay, Part I: Network Elements in Isolation. IEEE Transactions on Information Theory, 37(1):114-131, January 1991. 
[3] R. Cruz. A Calculus for Network Delay, Part II: Network Analysis. IEEE Transactions on Information Theory, 37(1):132-141, January 1991.

[4] D. Ferrari and D. Verma. A Scheme for RealTime Channel Establishment in Wide-Area Networks. IEEE Journal on Selected Areas in Communications, 8(3):368-379, April 1990.

[5] V. Frost and B. Melamed. Traffic modeling for telecommunications networks. IEEE Communication Magazine, 32(3):70-81, March 1994.

[6] D. Le Gall. MPEG: A Video Compression Standard for Multimedia Applications. Communications of the ACM, 34(4):305-313, April 1991.

[7] J. Hyman, A. Lazar, and G. Pacifici. Joint Scheduling and Admission Control for ATS-based Switching Nodes. In Proc. SIGCOMM '92, pages 223-234, August 1992 .

[8] E. Knightly and H. Zhang. Traffic characterization and switch utilization using deterministic bounding interval dependent traffic models. In Proc. IEEE INFOCOM'95, April 1995.

[9] J. Liebeherr, D. Wrege, and D. Ferrari. Exact Admission Control in Networks with Bounded Delay Services. To appear: IEEE/ACM Transactions on Networking.

[10] I. Nikolaidis and R. Onvural. A Bibliography on Performance Issues in ATM Networks. Computer Communications Review, 22(5):8-23, October 1992.

[11] A. Parekh and R. Gallager. A generalized processor sharing approach to flow control in integrated services networks: The multiple node case. In Proceedings of IEEE INFOCOM'93, pages 521-530, San Francisco, CA, March 1993.

[12] C. Partridge. Gigabit Networking. Addison-Wesley Publishing Company, 1994.

[13] K. Patel, B. Smith, and L. Rowe. Performance of a software MPEG video decoder. In Proceedings of the 1st ACM International Conference on Multimedia, pages 75-82, Anaheim, CA, August 1993.

[14] O. Rose. Statistical Properties of MPEG Video Traffic and Their Impact on Traffic Modeling in ATM Systems. Technical Report 101, Institute of Computer Science, University of Wurzburg, Germany, February 1995.

[15] J. Turner. New Directions in Communications (or Which Way to the Information Age?). IEEE Communications Magazine, 25(8):8-15, October 1986.

[16] H. Zhang and D. Ferrari. Rate-Controlled StaticPriority Queueing. In Proc. IEEE INFOCOM '93, pages 227-236, April 1993.

[17] H. Zhang and D. Ferrari. Improving Utilization for Deterministic Service in Multimedia Communication. In 1994 International Conference on Multimedia Computing and Systems, pages 295-304, May 1994.

[18] H. Zhang and D. Ferrari. Rate-controlled service disciplines. Journal of High Speed Networks, 3(4):389-412, 1994.
Dallas E. Wrege (S'93 - S'91) received the B.A. degree in Applied Mathematics from Harvard University in 1992.

He is currently a Research Assistant in the Multimedia Group at the Department of Computer Science, University of Virginia, where he is working toward the Ph.D. degree. His main research interests are in the area of multimedia and quality-of-service networks.

Edward W. Knightly (S '92) received the B.S. degree from Auburn University in 1991 and the M.S. degree from the University of California at Berkeley in 1992, both in Electrical Engineering. He expects to receive his $\mathrm{Ph}$.D. from the University of California at Berkeley in 1996.

His research interests are in the areas of high speed networks, realtime multimedia communication, resource management algorithms, and system performance analysis.

Hui Zhang (M'94 - ACM' 94) received the B.S. degree in Computer Science from Beijing University in 1988, the M.S. degree in Computer Engineering from Rensselaer Polytechnic Institute in 1989, and the Ph.D. degree in Computer Science from the University of California at Berkeley in 1993.

He is currently an assistant professor in the School of Computer Science at Carnegie Mellon University. His research interests are in integrated services networks and multimedia systems. His email address is: hzhang@cs.cmu.edu.

Jörg Liebeherr (S'88 - M'92) received the Diplom-Informatiker degree from the University of Erlangen, Germany, in 1988 and the $\mathrm{Ph} . \mathrm{D}$. degree in Computer Science from the Georgia Institute of Technology in 1991.

From 1990 until 1991 he did his dissertation work partly at the IBM T. J. Watson Research Center. In 1992 he was a Postdoctoral Fellow in the Computer Science Division of the University of California, Berkeley. Since September 1992 he is an Assistant Professor in the Department of Computer Science at the University of Virginia. His research interests are multimedia networks, broadband networks, real-time systems and performance evaluation. He is a member of IEEE and ACM (Sigcomm and Sigmetrics). 